einem "Versehen" der Mutter in die Schuhe. Was nun unsern Fall anbetrifft, so wäre es ein Wunder gewesen, wenn sich die Angehörigen und die Nachbarn die prächtige Gelegenheit, für diesen Riesenwuchs eỉn sogenanntes Versehen der Mutter verantwortlich zu machen, hätten ent-s gehen lassen. Der vorerwähnten Schwester des Vaters, welche den Riesenwuchs der großen rechten Zehe hatte, wurde nämlich diese Mißbildung operativ zu der Zeit entfernt, als die Mutter, die nichts von der Operation gewußt haben soll, mit unserem Patienten schwanger ging. Näheres konnte ich leider darüber nicht erfahren, da der Kollege, ein Dr. Hofmann, der die Operation ausgeführt hat, längst gestorben ist. Die amputierte große Zehe wurde der Mutter nach der Operation unerwartet gezeigt, worauf sie furchtbar erschrak, laut aufschrie und sich vor Angst in den rechten Daumen biß. Relata refero! Als nun das Kind mit Hilfe der Hebamme zur Welt gekommen war und der vergrößerte rechte Daumen bemerkt wurde, waren alle Angehörigen und die ganze Nachbarschaft die weise Frau natürlich an der Spitze - darüber völlig einig, daß dies die abgeschnittene rechte Zehe der Tante sei, die dem jungen Erdenbürger, anstatt des Daumens an die rechte Hand gewachsen wäre.

Der Besitzer dieses großen Daumens hat bis jetzt absolut keine Unzuträglichkeiten davon gehabt. Er ist Schreiner und freut sich, daß der vergrößerte Daumen nicht an der linken Hand sitzt, da er sonst die Nägel beim Einschlagen nicht gut halten könne. Beim Handhaben des Hammers, des Hobels, der Säge und anderer Berufsinstrumente hat er keine Unbequemlichkeiten.

\title{
2.
}

\section{Über einen Fall von kongenitalem Lymphosarkom des Pankreas.}

\author{
Von
}

Dr. Adrien L'Huillier, Assistenten am Pathologischen Institut zu Genf.

Verfossenes Jahr fand ich bei der Sektion eines Neugeborenen neben anderen ein primäres, offenbar kongenitales Sarkom im Pankreaskopf. $\mathrm{Da}$ ich in der mir zugänglichen Literatur einen gleichartigen Fall nicht auffinden konnte, erscheint er mir mitteilenswert.

Es betraf ein neun Tage altes Mädchen, welches am 12. Mai 1903 geboren wurde, und am 20. Mai infolge einer umbilikalen Infektion starb.

Der pathologisch-anatomische Befund war folgender.

Periumbilikales Geschwür, eitrige Phlebitis der Vena umbilicalis, Leberabsceß, eitrige Peritonitis, doppelseitige Bronchopnemnonie; akute Splenitis, Harmsäureinfarkte, geringgradige Nephritis parenchymatosa, erweiterte Ureteren. 
An Stelle des Pankreaskopfes fand ich eine Geschwulst von $32 \mathrm{~mm}$ Länge, $21 \mathrm{~mm}$ Breite, von ziemlich fester, elastischer Beschaffenheit und grauer Farbe. Stellenweise zeigte sich Gallenfärbung. Sie war mit dem Leberhilus, dem Duodenum und dem Zwerchfelle bindegewebig verwachsen. Der von ihr freie Pankreaskörper hatte ein makroskopisch normales Aussehen, war aber kleiner als derjenige gleichaltriger und gleichgroßer Kinder, wie ich mich durch Vergleichung mit solchen überzeugt habe. Die mikroskopische Untersuchang des Pankreaskörpers ergibt, dab das interalveoläre Bindegewebe stärker entwickelt ist, als es normalerweise sein sollte und anch eine derbere Beschaffenheit darbietet. Dies gilt besonders für das perikanalikuläre Bindegewebe. Hier und in den der Geschwulst zunächst gelegenen Partien besteht eine mehr oder minder starke Rundzelleninfiltration. Die Blutgefäßwandungen sind etwas verdickt, aber ohne sonstige Veränderungen.

Das im Pankreaskörper vorhandene Lymphoidgewebe verhält sich ganz ebenso wie die lymphatischen Knötchen im normalen Pankreas andrer Neugeborenen, wie ich mich durch mehrfache Vergleichung überzeugen konnte.

Was nun die Geschwulst selbst betrifft, so bestand dieselbe aus kleinen, runden, Lymphocyten ähnlichen, einkörnigen Zellen, die nirgends eine regressive Metamorphose erkennen ließen. Zwischen ihnen fand sich nur sehr spärliches retikuläres Bindegewebe. Stellenweise sah man außerdem noch große, scharf begrenzte Riesenzellen mit hellem durchsichtigen Protoplasma und zahlreichen in diesem zerstreut liegenden Kernen.

Die in der Geschwulst ziemlich zahlreichen Gefäße sind dünnwandig. In den kleinsten derselben fand ich da und dort aus Blutplättchen bestehende, gut färbbare Thromben. Auch in einigen größeren, meistens an der Peripherie liegenden Gefäßen sind teils aus Blutplättchen, teils aus Leukocyten bestehende 'Thromben vorhanden.

Der makroskopische und mikroskopische Befund an der Geschwulst und dem übrigen Pankreaskörper lassen erkennen, daß die hier gefundenen Veränderungen mit der eitrigen Umbilikalaffektion und ihren Folgen absolut nichts zu tun haben. Veränderungen jüngeren Datums sind an ihnen nirgends vorhanden, und selbst die bindegewebigen Adhäsionen zwischen der Geschwulst und ihrer Umgebung sind ihrer Beschaffenheit nach schon älter. Der Tumor ist seinem Bau nach zweifellos ein Lymphosarkom, und da er während der kurzen Lebensdauer des Kindes nicht entstanden und sich bis zur vorgefundenen Größe entwickelt haben kann, ist er offenbar ein kongenitales Lymphosarkom.

Dadurch ist dasselbe vielleicht einzig in seiner Art, denn der von Litten beobachtete und mitgeteilte Fall eines bei einem vierjährigen Kinde im Pankreas vorhandenen Rundzellensarkoms mit Metastasen im Ileum dürfte, wenn vielleicht auch lymphatischer Natur, kaum angeboren gewesen sein. ${ }^{1}$ )

1) Litten, Sarcome primitif du Pancreas. Séance de la soc. méd. de Berlin. Semaine médicale, Paris 1888. 
Das gleiche gilt für einen von Dr. Malherbe veröffentlichten Fall von Lymphosarkom des Pankreas, Duodenums und der anliegenden Lymphdrïsen, der sogar wahrscheinlich sekundär war. ${ }^{1}$ )

Ob letzteres bei einem von Lépine und Cornil veröffentlichten Falle von "Lymphosarcome du pancréas et de plusieurs autres organes" 3 ) sich ebenso verhielt, lasse ich dahingestellt. Ein eigentümlicher Befund war bei unserem Fall das Vorhandensein von Riesenzellen, doch ist dasselbe kein Unikum. Ich selbst hatte Gelegenheit, solche Gebilde in einem harten Lymphosarkom, welches im Jahre 1903 im mikroskopischen Kurs verteilt wurde, zu sehen und den Studenten zu zeigen. Sodann berichtet Piccoli über das Vorkommen von Riesenzellen in einem sekundären Rundzellensarkom des Pankreas. ${ }^{2}$ )

Schwer, oder vielmehr nicht zu entscheiden ist in unserem Fall die Frage, ob die Geschwulst von den im Pankreas vorhandenen lymphatischen. Gebilden oder von einem anderswoher kommenden und ins Pankreas verlagerten lymphatischen Keime ausging.

Eine ebenso schwer zu entscheidende Frage ist diejenige über die Entstehung der im Pankreasköper vorhandenen interstitiellen Veränderungen.

Die Möglichkeit, daß sie und vielleicht auch die Geschwulst selbst syphilitischer Natur sein könnten, mußte abgelehnt werden, da weder die Geschwulst, noch das interstitielle Gewebe des Pankreas einen für Syphilis sprechenden Bau hatten, da sonst nirgends syphilitische Veränderungen vorhanden waren, und endlich da die anamnestischen Daten ganz gegen eine solche Affektion sprachen.

Diese interstitiellen Veränderungen müssen somit als rein entzündliche Erscheinungen aufgefaßt werden, vielleicht verursacht durch Lymphstauung infolge der Geschwulstentwicklung im Pankreaskopf. Die perikanalikulären Lymphgefäße waren nämlich in der Tat deutlich erweitert, während dies bei den Drüsenkanälchen und Läppchen nicht der Fall war. Letzteres ist allerdings nicht wunderbar, da das Pankreas während des Foetallebens kaum sezernieren dürfte.

1) Malherbe, Tumeur du duodenum et du pancreas (lymphosarcome) in „Bulletins de la société anatomique de Nantes. Anné 1880, pag. 76.

2) Piccoli, Sarkom des Pankreas in Zieglers Beiträgen. Bd. XXII, 1. Heft, 1897, S. 105. 